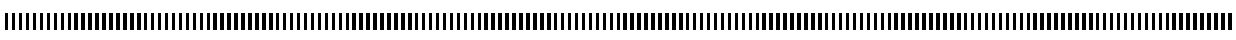

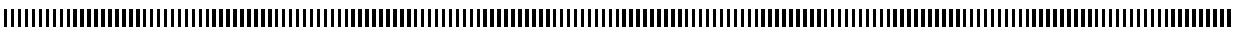

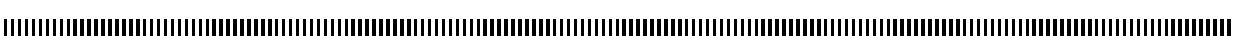

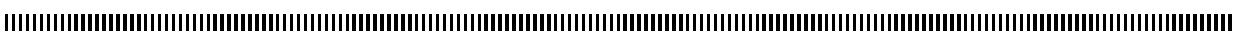

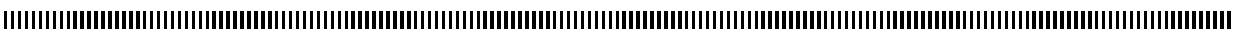

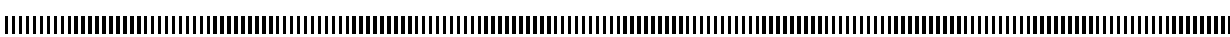

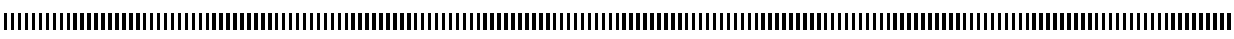

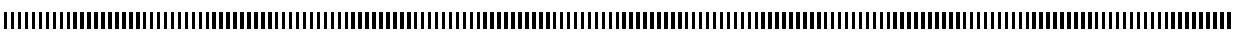

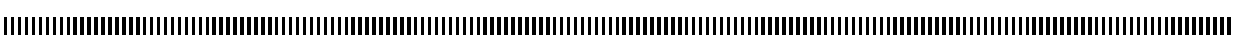
|||||||||||||||||||||||||||||||||||||||||||||||||||||||||||||||||||||||||||||||||||||||||||||||||||||||||||||||||||||||||||||||||||||||||||||||||||||||||||||||||||||||||||||||||||||||||||||||||||||||

\title{
Modélisation asymptotique d'une coque peu-profonde de Marguerre-von Kármán généralisée dans le cas dynamique
}

\author{
D.A. Chacha* - A. Ghezal ${ }^{* *}-$ A. Bensayah ${ }^{* * *}$ \\ * Département de Maths-infor, Université Kasdi Merbah, \\ B.P 511 Ouargla 30000, Algérie \\ d_chacha@hotmail.com \\ ** Département de Maths-infor, Université Kasdi Merbah, \\ B.P 511 Ouargla 30000, Algérie \\ ghezalark@hotmail.fr \\ *** Département de Maths-infor, Université Kasdi Merbah, \\ B.P 511 Ouargla 30000, Algérie \\ bensayahabd@gmail.com
}

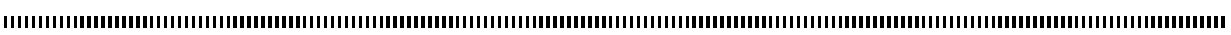

RÉSUMÉ. Dans un travail récent Gratie [7] a généralisé les équations de Marguerre-von Kármán classiques étudiées par Ciarlet et Paumier dans [2], où une partie seulement de la face latérale est soumise à des conditions aux limites de type von Kármán et la partie restante étant libre. Elle montre que le terme dominant du développement asymptotique est caractérisé par un problème aux limites bi-dimensionnel. Dans ce travail, on étend formellement cette étude au cas dynamique.

ABSTRACT. In a recent work Gratie [7] has generalized the classical Marguerre-von Kármán equations studied by Ciarlet and Paumier in [2], where only a portion of the lateral face is subjected to boundary conditions of von Kármán's type and the remaining portion being free. She shows that the leading term of the asymptotic expansion is characterized by a two-dimensional boundary value problem. In this paper, we extend formally this study to dynamic case.

MOTS-CLÉS : élasticité non linéaire, analyse asymptotique, coque peu-profonde, Marguerre-von Kármán, dynamique.

KEYWORDS : nonlinear elasticity, asymptotic analysis, shallow shell, Marguerre-von Kármán, dynamic.

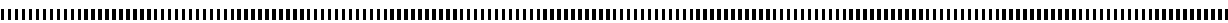




\section{Introduction}

Les équations classiques de Marguerre-von Kármán, qui constituent un modèle mathématique de l'équilibre d'une coque peu-profonde sous l'action des forces de pression horizontales, ont été proposées par Marguerre [16], et par von Kármán et Tsien [17]. En 1986 Ciarlet et Paumier [2] ont donné une justification mathématique à ces équations par l'analyse asymptotique. Ces équations en coordonnées curvilignes ont été justifiées par Andreoiu-Banica [5]. En 2002 Gratie [7] a généralisé ces équations, où une partie seulement de la face latérale est soumise à des conditions aux limites de type von Kármán et la partie restante étant libre, ensuite Ciarlet et Gratie $[9,8]$ ont établi un théorème d'existence pour ces équations, pour les deux modèles plaques de von Kármán généralisées et coques peu-profondes de Marguerre-von Kármán généralisées.

Dans le cadre de l'élasticité linéaire et dans le cas dynamique, une justification par l'analyse asymptotique des équations des coques minces (membranaire, en flexion et de Koiter) a été proposé par Xiao [11, 12,13], pour les coques membranaires généralisées par Ye [14] et pour les coques peu-profondes d'épaisseur variable par Yan [15].

Ce travail rentre dans le cadre de la modélisation asymptotique des structures minces, il généralise ainsi les travaux [7, 8] au cas dynamique et les travaux [11, 12,14] au cas non linéaire mais avec des conditions au bord latéral de type von Kármán généralisées.

\section{Le problème tri-dimensionnel}

Dans toute la suite de ce travail, on utilise les conventions et notations suivantes : les indices Latins prennent leurs valeurs dans l'ensemble $\{1,2,3\}$ tandis que les indices Grecs prennent leurs valeurs dans l'ensemble $\{1,2\}$. La convention de la sommation par rapport aux indices répétés est systématiquement utilisée. On note $\partial_{i}=\frac{\partial}{\partial x_{i}}, \partial_{i}^{\varepsilon}=\frac{\partial}{\partial x_{i}^{\varepsilon}}$, $\hat{\partial}_{i}^{\varepsilon}=\frac{\partial}{\partial \hat{x}_{i}^{\varepsilon}}$ et $\delta_{i j}$ le symbole de Kronecker.

On se donne un ouvert borné connexe $\omega$ du plan horizontal $\mathbb{R}^{2}$, localement d'un même côté de sa frontière $\gamma$, supposée Lipschitzienne et on suppose que $0 \in \gamma$ et on note par $\gamma(y)$ l'arc de $\gamma$ qui joint 0 et le point $y \in \gamma$. Soit $\gamma_{1}$ et $\gamma_{2}$ deux parties disjointes de $\gamma$ et relativement ouvertes dans $\omega$, telles que $\lg r \gamma_{1}>0, \lg r \gamma_{2}>0$ et $\lg r\left(\gamma-\left\{\gamma_{1} \cup \gamma_{2}\right\}\right)=0$. On note $\left(\nu_{\alpha}\right)$ le vecteur unitaire normal extérieur le long de $\gamma$ et $\left(\tau_{\alpha}\right)$ le vecteur unitaire tangent à $\gamma$ défini par $\tau_{1}=-\nu_{2}$ et $\tau_{2}=\nu_{1}$, on note $\partial_{\nu}=\nu_{\alpha} \partial_{\alpha}$ la dérivée normale extérieure, $\partial_{\tau}=\tau_{\alpha} \partial_{\alpha}$ la dérivée tangentielle dans la direction du vecteur $\left(\tau_{\alpha}\right)$ le long de $\gamma$. Pour $\varepsilon>0$, on pose $\left.\Omega^{\varepsilon}=\omega \times\right]-\varepsilon, \varepsilon\left[, \Gamma_{ \pm}^{\varepsilon}=\omega \times\{ \pm \varepsilon\}\right.$.

Soit $\theta^{\varepsilon} \in C^{3}(\bar{\omega})$ une fonction donnée qui satisfait $\theta^{\varepsilon}=\partial_{\nu} \theta^{\varepsilon}=0$ sur $\gamma_{1}$, la fonction $\theta^{\varepsilon}$ doit être d'ordre $\varepsilon$ pour que la coque soit faiblement courbée (voir [2]). On définit l'application $\Theta^{\varepsilon}: \bar{\Omega}^{\varepsilon} \rightarrow \mathbb{R}^{3}$ par $\Theta^{\varepsilon}\left(x^{\varepsilon}\right)=\left(x_{1}, x_{2}, \theta^{\varepsilon}\left(x_{1}, x_{2}\right)\right)+x_{3}^{\varepsilon} a_{3}^{\varepsilon}\left(x_{1}, x_{2}\right), a_{3}^{\varepsilon}$ le vecteur normal unitaire à la surface moyenne définie par $\Theta^{\varepsilon}(\bar{\omega} \times\{0\})$. On note $\hat{\Omega}^{\varepsilon}=$ 
$\Theta^{\varepsilon}\left(\Omega^{\varepsilon}\right), \hat{\gamma}_{1}^{\varepsilon}=\Theta^{\varepsilon}\left(\gamma_{1}\right)$ et $\hat{\Gamma}_{ \pm}^{\varepsilon}=\Theta^{\varepsilon}\left(\Gamma_{ \pm}^{\varepsilon}\right)$. Pour $\varepsilon$ suffisamment petit, $\Theta^{\varepsilon}: \bar{\Omega}^{\varepsilon} \rightarrow \Theta^{\varepsilon}\left(\bar{\Omega}^{\varepsilon}\right)$ est un $C^{1}$-difféomorphisme (voir [2, Proposition 3.2]).

On considère alors une coque peu-profonde non linéairement élastique dans le cas dynamique, de configuration de référence $\left\{\hat{\Omega}^{\varepsilon}\right\}^{-}$, formée d'un matériau de Saint-Venant Kirchhoff de constantes de Lamé $\lambda^{\varepsilon}>0$ et $\mu^{\varepsilon}>0$, soumise à des forces de volume de densité $\left(\hat{f}_{i}^{\varepsilon}\right)=\left(0,0, \hat{f}_{3}^{\varepsilon}\right)$ en son intérieur $\hat{\Omega}^{\varepsilon}$, à des forces de surface de densité $\left(\hat{g}_{i}^{\varepsilon}\right)=$ $\left(0,0, \hat{g}_{3}^{\varepsilon}\right)$ sur sa face supérieure $\hat{\Gamma}_{+}^{\varepsilon}$ et sa face inférieure $\hat{\Gamma}_{-}^{\varepsilon}$ et à des forces de pression horizontales $\left(\hat{h}_{1}^{\varepsilon}, \hat{h}_{2}^{\varepsilon}, 0\right)$ de type von Kármán au sens de [1] sur la partie $\Theta^{\varepsilon}\left(\gamma_{1} \times[-\varepsilon, \varepsilon]\right)$ de sa face latérale. Sur la partie restante $\Theta^{\varepsilon}\left(\gamma_{2} \times[-\varepsilon, \varepsilon]\right)$ de sa face latérale, la coque est soumise à des conditions aux limites de bord libre; $\left(\hat{n}_{i}^{\varepsilon}\right)$ est la normale extérieure unitaire le long de $\partial \hat{\Omega}^{\varepsilon}$, $\hat{\rho}^{\varepsilon}$ est la densité de masse, $\hat{p}^{\varepsilon}$ et $\hat{q}^{\varepsilon}$ sont des données, $\hat{\sigma}_{i j}^{\varepsilon}=$ $\lambda^{\varepsilon} \hat{E}_{p p}^{\varepsilon}\left(\hat{u}^{\varepsilon}\right) \delta_{i j}+2 \mu^{\varepsilon} \hat{E}_{i j}^{\varepsilon}\left(\hat{u}^{\varepsilon}\right)$ les composantes du tenseur des contraintes et $\hat{E}_{i j}^{\varepsilon}\left(\hat{u}^{\varepsilon}\right)=$ $\frac{1}{2}\left(\hat{\partial}_{i}^{\varepsilon} \hat{u}_{j}^{\varepsilon}+\hat{\partial}_{j}^{\varepsilon} \hat{u}_{i}^{\varepsilon}+\hat{\partial}_{i}^{\varepsilon} \hat{u}_{m}^{\varepsilon} \hat{\partial}_{j}^{\varepsilon} \hat{u}_{m}^{\varepsilon}\right)$ les composantes du tenseur des déformations.

Enfin on définit les espaces suivants :

$V\left(\hat{\Omega}^{\varepsilon}\right)=\left\{\begin{array}{c}\hat{v}^{\varepsilon}=\left(\hat{v}_{i}^{\varepsilon}\right) \in \boldsymbol{W}^{1,4}\left(\hat{\Omega}^{\varepsilon}\right) ; \hat{v}_{\alpha}^{\varepsilon} \text { indépendantes de } \hat{x}_{3}^{\varepsilon} \text { et } \hat{v}_{3}^{\varepsilon}=0 \\ \operatorname{sur} \Theta^{\varepsilon}\left(\gamma_{1} \times[-\varepsilon, \varepsilon]\right)\end{array}\right\}$,

$V\left(\Omega^{\varepsilon}\right)=\left\{\begin{array}{c}v^{\varepsilon}=\left(v_{i}^{\varepsilon}\right) \in \boldsymbol{W}^{1,4}\left(\Omega^{\varepsilon}\right) ; v_{\alpha}^{\varepsilon} \text { indépendantes de } x_{3}^{\varepsilon} \text { et } v_{3}^{\varepsilon}=0 \\ \operatorname{sur} \gamma_{1} \times[-\varepsilon, \varepsilon]\end{array}\right\}$,

$V(\Omega)=\left\{\begin{array}{c}v=\left(v_{i}\right) \in \boldsymbol{W}^{1,4}(\Omega) ; v_{\alpha} \text { indépendantes de } x_{3} \text { et } v_{3}=0 \\ \text { sur } \gamma_{1} \times[-1,1]\end{array}\right\}$,

$V_{K L}(\Omega)=\left\{\begin{array}{c}v=\left(v_{i}\right) \in \boldsymbol{H}^{1}(\Omega) ; v_{\alpha} \text { indépendantes de } x_{3} \text { et } v_{3}=0 \\ \text { sur } \gamma_{1} \times[-1,1], \partial_{i} v_{3}+\partial_{3} v_{i}=0 \text { dans } \Omega\end{array}\right\}$,

$V(\omega)=\left\{\eta=\left(\eta_{i}\right) \in H^{1}(\omega) \times H^{1}(\omega) \times H^{2}(\omega) ; \eta_{3}=\partial_{\nu} \eta_{3}=0\right.$ sur $\left.\gamma_{1}\right\}$,

$\boldsymbol{L}_{s}^{2}\left(\Omega^{\varepsilon}\right)=\left\{\tau^{\varepsilon}=\left(\tau_{i j}^{\varepsilon}\right) \in \boldsymbol{L}^{2}\left(\Omega^{\varepsilon}\right) ; \tau_{i j}^{\varepsilon}=\tau_{j i}^{\varepsilon}\right\}$.

Le déplacement inconnu $\hat{u}^{\varepsilon}=\left(\hat{u}_{i}^{\varepsilon}\right)\left(\hat{x}^{\varepsilon}, t\right)$ est solution du problème aux limites suivant :

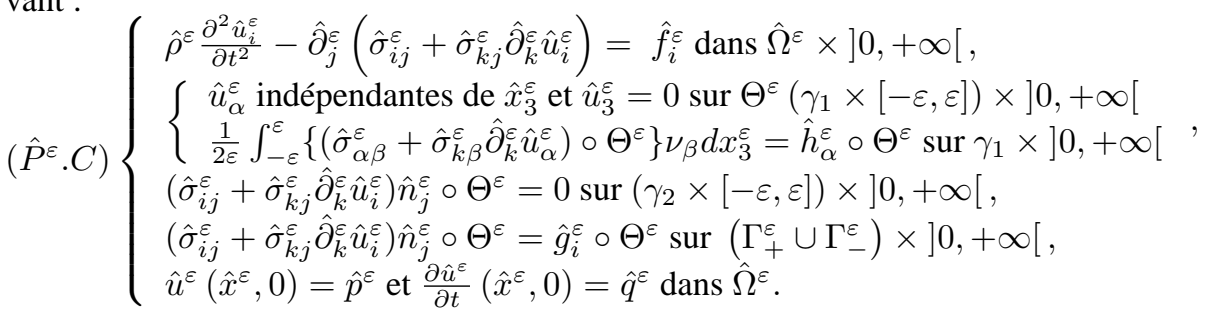

La formulation variationnelle du problème $\left(\hat{P}^{\varepsilon} . C\right)$ est :

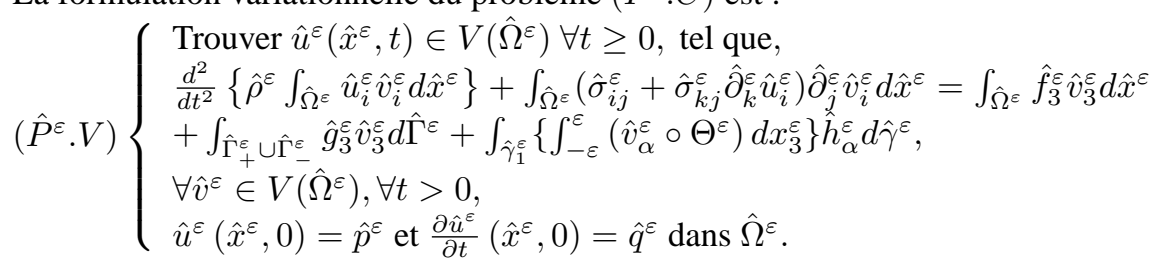


Proposition 1 Si $\Theta^{\varepsilon}$ est un $C^{1}$-difféomorphisme qui préserve l'orientation, alors le problème $\left(\hat{P}^{\varepsilon} . V\right)$ est équivaut à :

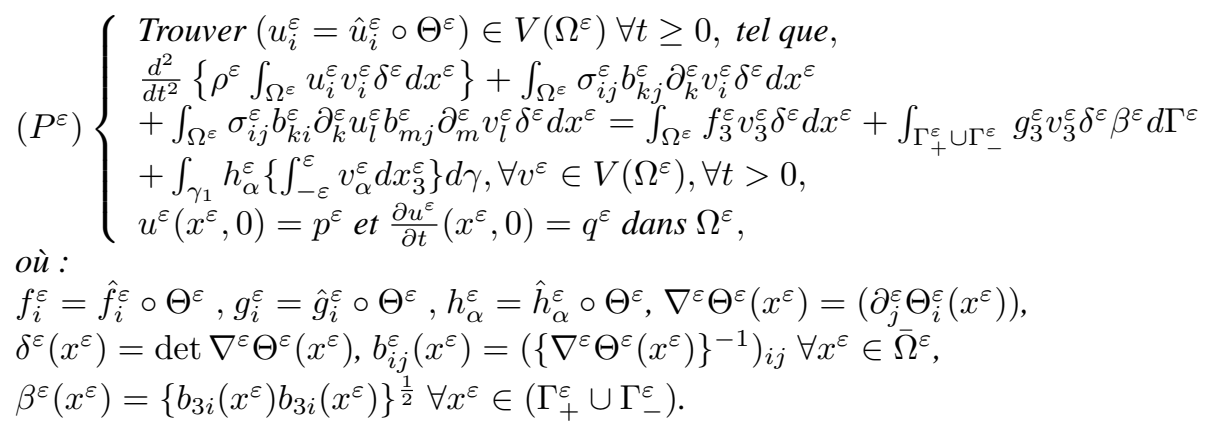

\section{Preuve.}

Dans le problème $\left(\hat{P}^{\varepsilon} \cdot V\right)$, en utilisant les relations suivantes : $\hat{\partial}_{j}^{\varepsilon} \hat{v}_{i}^{\varepsilon}=b_{k j}^{\varepsilon}\left(x^{\varepsilon}\right) \partial_{k}^{\varepsilon} v_{i}^{\varepsilon}\left(x^{\varepsilon}\right), d \hat{x}^{\varepsilon}=\delta^{\varepsilon} d x^{\varepsilon}, d \hat{\Gamma}^{\varepsilon}=\delta^{\varepsilon} \beta^{\varepsilon} d \Gamma^{\varepsilon}$, on obtient le problème $\left(P^{\varepsilon}\right)$.

\section{Application de la méthode des développements asymptotiques formels}

On commence par une mise à l'échelle du problème tri-dimensionnel, il s'agit de formuler le problème $\left(P^{\varepsilon}\right)$ sur un ouvert $\Omega$, indépendant de $\varepsilon$. Pour cela, on pose $\Omega=$ $\omega \times]-1,1\left[, \Gamma_{ \pm}=\omega \times\{ \pm 1\}\right.$, et à chaque point $x \in \bar{\Omega}$, on fait correspondre un point $x^{\varepsilon} \in \bar{\Omega}^{\varepsilon}$ par la bijection $\pi^{\varepsilon}: x=\left(x_{1}, x_{2}, x_{3}\right) \in \bar{\Omega} \rightarrow x^{\varepsilon}=\left(x_{1}, x_{2}, \varepsilon x_{3}\right) \in \bar{\Omega}^{\varepsilon}$.

On associe aux fonctions $u^{\varepsilon}, v^{\varepsilon} \in V\left(\Omega^{\varepsilon}\right)$ et $\sigma^{\varepsilon} \in \boldsymbol{L}_{s}^{2}\left(\Omega^{\varepsilon}\right)$ les fonctions mises à l'échelle $u(\varepsilon), v$ et $\sigma(\varepsilon)$ définies par :

$\left\{\begin{array}{l}u_{\alpha}^{\varepsilon}\left(x^{\varepsilon}, t\right)=\varepsilon^{2} u_{\alpha}(\varepsilon)(x, t), u_{3}^{\varepsilon}\left(x^{\varepsilon}, t\right)=\varepsilon u_{3}(\varepsilon)(x, t), \\ v_{\alpha}^{\varepsilon}\left(x^{\varepsilon}\right)=\varepsilon^{2} v_{\alpha}(x), v_{3}^{\varepsilon}\left(x^{\varepsilon}\right)=\varepsilon v_{3}(x), \sigma_{\alpha \beta}^{\varepsilon}\left(x^{\varepsilon}, t\right)=\varepsilon^{2} \sigma_{\alpha \beta}(\varepsilon)(x, t), \\ \sigma_{\alpha 3}^{\varepsilon}\left(x^{\varepsilon}, t\right)=\varepsilon^{3} \sigma_{\alpha 3}(\varepsilon)(x, t), \sigma_{33}^{\varepsilon}\left(x^{\varepsilon}, t\right)=\varepsilon^{4} \sigma_{33}(\varepsilon)(x, t),\end{array}\right.$

pour tout $x^{\varepsilon}=\pi^{\varepsilon} x \in \bar{\Omega}^{\varepsilon}$. On fait des hypothèses essentielles sur les données :

il existe des constantes $\lambda>0, \mu>0, \rho>0$ et des fonctions $f_{3} \in L^{2}\left(0, T ; L^{2}(\Omega)\right)$, $g_{3} \in L^{2}\left(0, T ; L^{2}\left(\Gamma_{+} \cup \Gamma_{-}\right)\right), h_{\alpha} \in L^{2}\left(0, T ; L^{2}\left(\gamma_{1}\right)\right)$ (T fini), $\theta \in C^{3}(\bar{\omega})$ indépendantes de $\varepsilon$ et $p(\varepsilon) \in V(\Omega), q(\varepsilon) \in \boldsymbol{L}^{2}(\Omega)$ telles que :

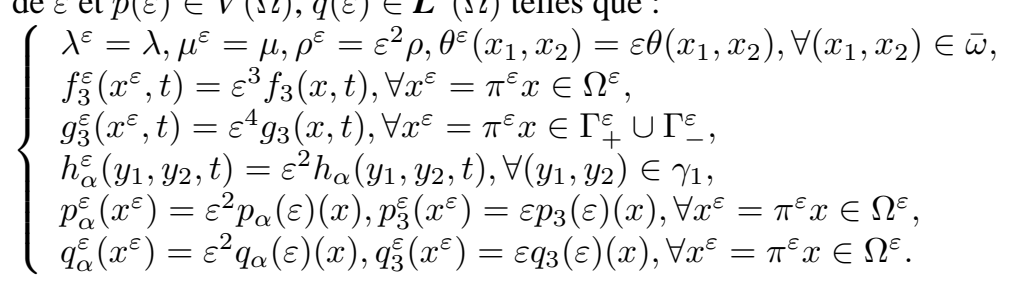


On obtient alors un problème $(P(\varepsilon))$ associé, posé sur l'ouvert $\Omega$.

Théorème 1 On suppose que $f_{3}^{\varepsilon}, g_{3}^{\varepsilon}, h_{\alpha}^{\varepsilon}$ et $\theta^{\varepsilon}$ vérifient les hypothèses précédentes, alors le problème $\left(P^{\varepsilon}\right)$ est équivaut à :

$$
(P(\varepsilon))\left\{\begin{array}{l}
\text { Trouver } u(\varepsilon)(x, t) \in V(\Omega) \forall t \in[0, T], \text { tel que, } \\
A^{t}(u(\varepsilon), v)+B^{\theta}(\sigma(\varepsilon), v)+2 C^{\theta}(\sigma(\varepsilon), u(\varepsilon), v)=F(v) \\
\left.+\varepsilon^{2} R(\varepsilon ; \sigma(\varepsilon), u(\varepsilon), v), \forall v \in V(\Omega), \forall t \in\right] 0, T[ \\
u(\varepsilon)(x, 0)=p(\varepsilon) \text { et } \frac{\partial u(\varepsilon)}{\partial t}(x, 0)=q(\varepsilon) \text { dans } \Omega,
\end{array}\right.
$$

où :

$A^{t}(u(\varepsilon), v)=-\frac{d^{2}}{d t^{2}}\left\{\rho \int_{\Omega} u_{3}(\varepsilon) v_{3} d x\right\}, B^{\theta}(\sigma(\varepsilon), v)=-\int_{\Omega} \sigma_{i j}(\varepsilon) \gamma_{i j}^{\theta}(v) d x$,

$C^{\theta}(\sigma(\varepsilon), u(\varepsilon), v)=-\frac{1}{2} \int_{\Omega} \sigma_{i j}(\varepsilon) \partial_{i}^{\theta} u_{3}(\varepsilon) \partial_{j}^{\theta} v_{3} d x$,

$F(v)=-\int_{\Omega} f_{3} v_{3} d x-\int_{\Gamma_{+} \cup \Gamma_{-}} g_{3} v_{3} d \Gamma-\int_{\gamma_{1}} h_{\alpha}\left\{\int_{-1}^{1} v_{\alpha} d x_{3}\right\} d \gamma$,

$\partial_{\alpha}^{\theta} v=\partial_{\alpha} v-\partial_{\alpha} \theta \partial_{3} v, \partial_{3}^{\theta} v=\partial_{3} v, \gamma_{i j}^{\theta}(v)=\frac{1}{2}\left(\partial_{i}^{\theta} v_{j}+\partial_{j}^{\theta} v_{i}\right)$.

\section{Preuve.}

La démonstration est similaire à celle du théorème 3.1 dans [2],

on a :

$$
\begin{gathered}
\int_{\Omega^{\varepsilon}} \sigma_{i j}^{\varepsilon} b_{k j}^{\varepsilon} \partial_{k}^{\varepsilon} v_{i}^{\varepsilon} \delta^{\varepsilon} d x^{\varepsilon}=\varepsilon^{5} \int_{\Omega} \sigma_{i j}(\varepsilon) \gamma_{i j}^{\theta}(v) d x+\varepsilon^{7} \varrho_{B}(\varepsilon ; \sigma(\varepsilon), v), \\
\int_{\Omega^{\varepsilon}} \sigma_{i j}^{\varepsilon} b_{k i}^{\varepsilon} \partial_{k}^{\varepsilon} u_{l}^{\varepsilon} b_{m j}^{\varepsilon} \partial_{m}^{\varepsilon} v_{l}^{\varepsilon} \delta^{\varepsilon} d x^{\varepsilon}=\varepsilon^{5} \int_{\Omega} \sigma_{i j}(\varepsilon) \partial_{i}^{\theta} u_{3}(\varepsilon) \partial_{j}^{\theta} v_{3} d x \\
+\varepsilon^{7} \varrho_{C}(\varepsilon ; \sigma(\varepsilon), u(\varepsilon), v), \\
\int_{\Omega^{\varepsilon}} f_{3}^{\varepsilon} v_{3}^{\varepsilon} \delta^{\varepsilon} d x^{\varepsilon}+\int_{\Gamma_{+}^{\varepsilon} \cup \Gamma_{-}^{\varepsilon}} g_{3}^{\varepsilon} v_{3}^{\varepsilon} \delta^{\varepsilon} \beta^{\varepsilon} d \Gamma^{\varepsilon}+\int_{\gamma_{1}} h_{\alpha}^{\varepsilon}\left\{\int_{-\varepsilon}^{\varepsilon} v_{\alpha}^{\varepsilon} d x_{3}^{\varepsilon}\right\} d \gamma= \\
\varepsilon^{5}\left(\int_{\Omega} f_{3} v_{3} d x+\int_{\Gamma_{+} \cup \Gamma_{-}} g_{3} v_{3} d \Gamma+\int_{\gamma_{1}} h_{\alpha}\left\{\int_{-1}^{1} v_{\alpha} d x_{3}\right\} d \gamma\right)+\varepsilon^{7} \varrho_{F}(\varepsilon ; v) .
\end{gathered}
$$

De plus :

$$
\frac{d^{2}}{d t^{2}}\left\{\rho^{\varepsilon} \int_{\Omega^{\varepsilon}} u_{i}^{\varepsilon} v_{i}^{\varepsilon} \delta^{\varepsilon} d x^{\varepsilon}\right\}=\varepsilon^{5} \frac{d^{2}}{d t^{2}}\left\{\rho \int_{\Omega} u_{3}(\varepsilon) v_{3} d x\right\}+\varepsilon^{7} \varrho_{A}(\varepsilon ; u(\varepsilon), v) .
$$

Alors :

$$
\begin{array}{r}
-\frac{d^{2}}{d t^{2}}\left\{\rho \int_{\Omega} u_{3}(\varepsilon) v_{3} d x\right\}-\int_{\Omega} \sigma_{i j}(\varepsilon) \gamma_{i j}^{\theta}(v) d x-\int_{\Omega} \sigma_{i j}(\varepsilon) \partial_{i}^{\theta} u_{3}(\varepsilon) \partial_{j}^{\theta} v_{3} d x= \\
-\int_{\Omega} f_{3} v_{3} d x-\int_{\Gamma_{+} \cup \Gamma_{-}} g_{3} v_{3} d \Gamma-\int_{\gamma_{1}} h_{\alpha}\left\{\int_{-1}^{1} v_{\alpha} d x_{3}\right\} d \gamma+\varepsilon^{2} R(\varepsilon ; \sigma(\varepsilon), u(\varepsilon), v) .
\end{array}
$$


Les fonctions $\varrho_{A}, \varrho_{B}, \varrho_{C}$ et $\varrho_{F}$ sont des restes bornés pour $0 \leq \varepsilon \leq \varepsilon_{0}$, leurs expressions ne sont pas nécessaires (voir par exemple [2]).

On applique les techniques de l'analyse asymptotique employé par Ciarlet [3] au problème $(P(\varepsilon))$ ( $\varepsilon$ un petit paramètre destiné à tendre vers zéro), on obtient le :

Théorème 2 On suppose que :

$$
(u(\varepsilon), \sigma(\varepsilon))=\left(u^{0}, \sigma^{0}\right)+\varepsilon\left(u^{1}, \sigma^{1}\right)+\varepsilon^{2}\left(u^{2}, \sigma^{2}\right)+\cdots,
$$

avec :

$$
\begin{gathered}
u^{0}=\left(u_{i}^{0}\right) \in V(\Omega), \partial_{3} u_{3}^{0} \in C^{0}(\bar{\Omega}), \\
u^{p}=\left(u_{i}^{p}\right) \in \boldsymbol{W}^{1,4}(\Omega), \forall p \geq 1, \sigma_{i j}^{0}=\sigma_{j i}^{0} \in L^{2}(\Omega) .
\end{gathered}
$$

Alors $u^{0}$ est de type Kirchhoff-Love et vérifie le problème suivant :

$$
\begin{aligned}
& \left(P_{K L}\right)\left\{\begin{array}{l}
\text { Trouver } u^{0} \in V_{K L}(\Omega) \forall t \in[0, T], \text { tel que, } \\
\frac{d^{2}}{d t^{2}}\left\{\rho \int_{\Omega} u_{3}^{0} v_{3} d x\right\}+\int_{\Omega} \sigma_{\alpha \beta}^{0} \partial_{\beta} v_{\alpha} d x+\int_{\Omega} \sigma_{\alpha \beta}^{0} \partial_{\alpha}\left(u_{3}^{0}+\theta\right) \partial_{\beta} v_{3} d x= \\
\left.\int_{\Omega} f_{3} v_{3} d x+\int_{\Gamma_{+} \cup \Gamma_{-}} g_{3} v_{3} d \Gamma+2 \int_{\gamma_{1}} h_{\alpha} v_{\alpha} d \gamma, \forall v \in V_{K L}(\Omega), \forall t \in\right] 0, T[, \\
u^{0}(x, 0)=p^{0} \text { et } \frac{\partial u^{0}}{\partial t}(x, 0)=q^{0} \text { dans } \Omega,
\end{array}\right. \\
& \text { où : } \\
& \sigma_{\alpha \beta}^{0}=\frac{2 \lambda \mu}{\lambda+2 \mu} \bar{E}_{\sigma \sigma}^{0}\left(u^{0}\right) \delta_{\alpha \beta}+2 \mu \bar{E}_{\alpha \beta}^{0}\left(u^{0}\right), \\
& \bar{E}_{\alpha \beta}^{0}\left(u^{0}\right)=\frac{1}{2}\left(\partial_{\alpha} u_{\beta}^{0}+\partial_{\beta} u_{\alpha}^{0}+\partial_{\alpha} u_{3}^{0} \partial_{\beta} u_{3}^{0}+\partial_{\alpha} \theta \partial_{\beta} u_{3}^{0}+\partial_{\beta} \theta \partial_{\alpha} u_{3}^{0}\right) .
\end{aligned}
$$

\section{Preuve.}

On introduit l'expression du développement asymptotique formel de $(u(\varepsilon), \sigma(\varepsilon))$ dans les équations du problème $(P(\varepsilon))$, puis on annule les coefficients des puissances successives de $\varepsilon$ trouvées dans ces équations, jusqu'à l'identification du terme d'ordre $\varepsilon^{0}$. Après une suite assez longue de calculs (des calculs semblables dans le cas statique sont détaillés dans $[3,4])$ on obtient le problème bi-dimensionnel $\left(P_{K L}\right)$.

On note que $u^{0}$ est de type Kirchhoff-Love, au sens $\partial_{i} u_{3}^{0}+\partial_{3} u_{i}^{0}=0$ dans $\Omega$.

\section{4. équivalence du premier terme du développement asymptotique avec la solution d'un modèle déplacement bi-dimensionnel}

On montre alors que le premier terme $u^{0}$ du développement asymptotique de $u(\varepsilon)$ peut-être complètement identifié à partir de la solution d'un problème variationnel bidimensionnel en déplacement, en ce sens que l'inconnue $\zeta$ qui y apparaît est le champ de 
déplacements des points de la surface moyenne $\bar{\omega}$ de la coque. De façon plus précise, on a le :

Théorème 3 Le terme $u^{0}=\left(u_{i}^{0}\right)$ est de la forme : $u_{\alpha}^{0}=\zeta_{\alpha}-x_{3} \partial_{\alpha} \zeta_{3}$ et $u_{3}^{0}=\zeta_{3}$, avec $\zeta=\left(\zeta_{i}\right) \in V(\omega)$ est la solution du problème variationnel suivant :

$(P(\omega))\left\{\begin{array}{l}2 \rho \int_{\omega} \frac{\partial^{2} \zeta_{3}}{\partial t^{2}} \eta_{3} d \omega-\int_{\omega} m_{\alpha \beta} \partial_{\alpha \beta} \eta_{3} d \omega+\int_{\omega} \bar{N}_{\alpha \beta} \partial_{\alpha}\left(\zeta_{3}+\theta\right) \partial_{\beta} \eta_{3} d \omega \\ \left.+\int_{\omega} N_{\alpha \beta} \partial_{\beta} \eta_{\alpha} d \omega=\int_{\omega} p_{3} \eta_{3} d \omega+2 \int_{\gamma_{1}} h_{\alpha} \eta_{\alpha} d \gamma, \forall \eta \in V(\omega), \forall t \in\right] 0, T[ \\ \zeta_{3}(., 0)=p_{3}^{0} \text { et } \frac{\partial \zeta_{3}}{\partial t}(., 0)=q_{3}^{0} \text { dans } \omega,\end{array}\right.$

où :

$m_{\alpha \beta}=-\frac{1}{3}\left\{\frac{4 \lambda \mu}{\lambda+2 \mu} \Delta \zeta_{3} \delta_{\alpha \beta}+4 \mu \partial_{\alpha \beta} \zeta_{3}\right\}$,

$\bar{N}_{\alpha \beta}=\frac{4 \lambda \mu}{\lambda+2 \mu} \bar{E}_{\sigma \sigma}^{0}(\zeta) \delta_{\alpha \beta}+4 \mu \bar{E}_{\alpha \beta}^{0}(\zeta)$,

$\bar{E}_{\alpha \beta}^{0}(\zeta)=\frac{1}{2}\left(\partial_{\alpha} \zeta_{\beta}+\partial_{\beta} \zeta_{\alpha}+\partial_{\alpha} \theta \partial_{\beta} \zeta_{3}+\partial_{\beta} \theta \partial_{\alpha} \zeta_{3}+\partial_{\alpha} \zeta_{3} \partial_{\beta} \zeta_{3}\right)$,

$p_{3}=\int_{-1}^{1} f_{3} d x_{3}+g_{3}(.,+1)+g_{3}(.,-1)$.

\section{Preuve.}

Il est bien connu (voir [4, Théorème 1.4-4]) que $v=\left(v_{i}\right) \in V_{K L}(\Omega)$ si et seulement s'il existe $\eta=\left(\eta_{i}\right) \in V(\omega)$, tel que $v_{\alpha}=\eta_{\alpha}-x_{3} \partial_{\alpha} \eta_{3}$ et $v_{3}=\eta_{3}$. Comme $v \in V_{K L}(\Omega)$, donc $v=\left(\eta_{1}-x_{3} \partial_{1} \eta_{3}, \eta_{2}-x_{3} \partial_{2} \eta_{3}, \eta_{3}\right)$, avec $\eta_{3} \in H^{2}(\omega)$ et $\eta_{3}=\partial_{\nu} \eta_{3}=0$ sur $\gamma_{1}$.

(i) On prend $v=\left(-x_{3} \partial_{1} \eta_{3},-x_{3} \partial_{2} \eta_{3}, \eta_{3}\right)$, avec $\eta_{3} \in H^{2}(\omega)$ et $\eta_{3}=\partial_{\nu} \eta_{3}=0$ sur $\gamma_{1}$, on déduit que :

$$
\begin{aligned}
\frac{d^{2}}{d t^{2}}\left\{\rho \int_{\Omega} \zeta_{3} \eta_{3} d x\right\}+\int_{\Omega}-x_{3} \sigma_{\alpha \beta}^{0} \partial_{\alpha \beta} \eta_{3} d x+ & \int_{\Omega} \sigma_{\alpha \beta}^{0} \partial_{\alpha}\left(\zeta_{3}+\theta\right) \partial_{\beta} \eta_{3} d x= \\
& \int_{\Omega} f_{3} \eta_{3} d x+\int_{\Gamma_{+} \cup \Gamma_{-}} g_{3} \eta_{3} d \Gamma
\end{aligned}
$$

(ii) On prend $v=\left(\eta_{1}, \eta_{2}, 0\right)$, avec $\eta_{\alpha} \in H^{1}(\omega)$, on déduit que :

$$
\int_{\Omega} \sigma_{\alpha \beta}^{0} \partial_{\beta} \eta_{\alpha} d x=2 \int_{\gamma_{1}} h_{\alpha} \eta_{\alpha} d \gamma .
$$

(iii) On applique la formule de Fubini : $\int_{\Omega} F d x=\int_{\omega}\left\{\int_{-1}^{1} F d x_{3}\right\} d \omega$, on obtient :

$$
\begin{aligned}
\frac{d^{2}}{d t^{2}}\left\{\rho \int_{\Omega} \zeta_{3} \eta_{3} d x\right\} & =2 \rho \int_{\omega} \frac{\partial^{2} \zeta_{3}}{\partial t^{2}} \eta_{3} d \omega, \\
\int_{\Omega}-x_{3} \sigma_{\alpha \beta}^{0} \partial_{\alpha \beta} \eta_{3} d x & =-\int_{\omega} m_{\alpha \beta} \partial_{\alpha \beta} \eta_{3} d \omega,
\end{aligned}
$$




$$
\begin{gathered}
\int_{\Omega} \sigma_{\alpha \beta}^{0} \partial_{\alpha}\left(\zeta_{3}+\theta\right) \partial_{\beta} \eta_{3} d x=\int_{\omega} \bar{N}_{\alpha \beta} \partial_{\alpha}\left(\zeta_{3}+\theta\right) \partial_{\beta} \eta_{3} d \omega \\
\int_{\Omega} f_{3} \eta_{3} d x+\int_{\Gamma_{+} \cup \Gamma_{-}} g_{3} \eta_{3} d \Gamma=\int_{\omega}\left\{\int_{-1}^{1} f_{3} d x_{3}+g_{3}(.,+1)+g_{3}(.,-1) \eta_{3} d \omega\right. \\
\int_{\Omega} \sigma_{\alpha \beta}^{0} \partial_{\beta} \eta_{\alpha} d x=\int_{\omega} \bar{N}_{\alpha \beta} \partial_{\beta} \eta_{\alpha} d \omega=2 \int_{\gamma_{1}} h_{\alpha} \eta_{\alpha} d \gamma .
\end{gathered}
$$

Alors :

$$
\begin{array}{r}
2 \rho \int_{\omega} \frac{\partial^{2} \zeta_{3}}{\partial t^{2}} \eta_{3} d \omega-\int_{\omega} m_{\alpha \beta} \partial_{\alpha \beta} \eta_{3} d \omega+\int_{\omega} \bar{N}_{\alpha \beta} \partial_{\alpha}\left(\zeta_{3}+\theta\right) \partial_{\beta} \eta_{3} d \omega \\
+\int_{\omega} \bar{N}_{\alpha \beta} \partial_{\beta} \eta_{\alpha} d \omega=\int_{\omega}\left\{\int_{-1}^{1} f_{3} d x_{3}+g_{3}(.,+1)+g_{3}(.,-1)\right\} \eta_{3} d \omega+2 \int_{\gamma_{1}} h_{\alpha} \eta_{\alpha} d \gamma .
\end{array}
$$

Il reste enfin à écrire le problème aux limites résolu, c'est-à-dire lorsque les données et la solution sont suffisamment régulières. On utilise les formules de Green ad hoc, on établit ensuite l'équivalence formelle du problème variationnel $(P(\omega))$ avec un problème aux limites en déplacement :

Théorème 4 Une solution suffisamment régulière $\zeta=\left(\zeta_{i}\right)$ du problème $(P(\omega))$ est la solution du problème suivant :

$$
(\bar{P}(\omega))\left\{\begin{array}{l}
\text { Trouver } \zeta \in V(\omega) \forall t \in[0, T], \text { tel que, } \\
\left.2 \rho \frac{\partial^{2} \zeta_{3}}{\partial t^{2}}-\partial_{\alpha \beta} m_{\alpha \beta}-\bar{N}_{\alpha \beta} \partial_{\alpha \beta}\left(\zeta_{3}+\theta\right)=p_{3} \text { dans } \omega \times\right] 0, T[ \\
\left.\partial_{\beta} N_{\alpha \beta}=0 \text { dans } \omega \times\right] 0, T[ \\
\left.\zeta_{3}=\partial_{\nu} \zeta_{3}=0 \text { sur } \gamma_{1} \times\right] 0, T[ \\
\left.\bar{N}_{\alpha \beta} \nu_{\beta}=2 h_{\alpha} \text { sur } \gamma_{1} \times\right] 0, T[ \\
\left.m_{\alpha \beta} \nu_{\alpha} \nu_{\beta}=0 \text { sur } \gamma_{2} \times\right] 0, T[ \\
\left.\partial_{\alpha} m_{\alpha \beta} \nu_{\beta}+\partial_{\tau}\left(m_{\alpha \beta} \nu_{\alpha} \tau_{\beta}\right)=0 \text { sur } \gamma_{2} \times\right] 0, T[ \\
\left.\bar{N}_{\alpha \beta} \nu_{\beta}=0 \text { sur } \gamma_{2} \times\right] 0, T[ \\
\zeta_{3}(., 0)=p_{3}^{0} \text { et } \frac{\partial \zeta_{3}}{\partial t}(., 0)=q_{3}^{0} \text { dans } \omega .
\end{array}\right.
$$

\section{Preuve.}

On applique la formule de Green, on trouve :

$$
\begin{aligned}
-\int_{\omega} m_{\alpha \beta} \partial_{\alpha \beta} \eta_{3} d \omega & =\int_{\gamma}\left\{\left(\partial_{\alpha} m_{\alpha \beta}\right) \nu_{\beta}+\partial_{\tau}\left(m_{\alpha \beta} \nu_{\alpha} \tau_{\beta}\right)\right\} \eta_{3} d \gamma \\
& -\int_{\gamma} m_{\alpha \beta} \nu_{\alpha} \nu_{\beta} \partial_{\nu} \eta_{3} d \gamma-\int_{\omega}\left(\partial_{\alpha \beta} m_{\alpha \beta}\right) \eta_{3} d \omega,
\end{aligned}
$$




$$
\begin{aligned}
& \int_{\omega} \bar{N}_{\alpha \beta} \partial_{\alpha}\left(\zeta_{3}+\theta\right) \partial_{\beta} \eta_{3} d \omega=-\int_{\omega}\left\{\partial_{\beta}\left(\bar{N}_{\alpha \beta} \partial_{\alpha}\left(\zeta_{3}+\theta\right)\right)\right\} \eta_{3} d \omega \\
&+\int_{\gamma}\left(\bar{N}_{\alpha \beta} \partial_{\alpha}\left(\zeta_{3}+\theta\right)\right) \nu_{\beta} \eta_{3} d \gamma \\
& \int_{\omega} \bar{N}_{\alpha \beta} \partial_{\beta} \eta_{\alpha} d \omega=-\int_{\omega}\left(\partial_{\beta} \bar{N}_{\alpha \beta}\right) \eta_{\alpha} d \omega+\int_{\gamma} \bar{N}_{\alpha \beta} \nu_{\beta} \eta_{\alpha} d \gamma .
\end{aligned}
$$

Donc :

$$
\begin{gathered}
\int_{\omega}\left[2 \rho \frac{\partial^{2} \zeta_{3}}{\partial t^{2}}-\partial_{\alpha \beta} m_{\alpha \beta}-\partial_{\beta}\left(\bar{N}_{\alpha \beta} \partial_{\alpha}\left(\zeta_{3}+\theta\right)\right)-p_{3}\right] \eta_{3} d \omega- \\
\int_{\omega}\left(\partial_{\beta} \bar{N}_{\alpha \beta}\right) \eta_{\alpha} d \omega+\int_{\gamma}\left(\bar{N}_{\alpha \beta} \nu_{\beta}-2 \tilde{h}_{\alpha}\right) \eta_{\alpha} d \gamma-\int_{\gamma_{2}} m_{\alpha \beta} \nu_{\alpha} \nu_{\beta} \partial_{\nu} \eta_{3} d \gamma+ \\
\int_{\gamma_{2}}\left\{\left[\partial_{\alpha} m_{\alpha \beta}+\bar{N}_{\alpha \beta} \partial_{\alpha}\left(\zeta_{3}+\theta\right)\right] \nu_{\beta}+\partial_{\tau}\left(m_{\alpha \beta} \nu_{\alpha} \tau_{\beta}\right)\right\} \eta_{3} d \gamma=0,
\end{gathered}
$$

pour tout $\eta=\left(\eta_{\alpha}, \eta_{3}\right) \in V(\omega)$. Les fonctions $\tilde{h}_{\alpha}: \gamma \times[0, T] \rightarrow \mathbb{R}$ définies par :

$$
\tilde{h}_{\alpha}=h_{\alpha} \text { sur } \gamma_{1} \times[0, T] \text { et } \tilde{h}_{\alpha}=0 \text { sur } \gamma_{2} \times[0, T] .
$$

L'équation précédente implique que tous les coefficients de $\eta_{\alpha}, \eta_{3}$, et $\partial_{\nu} \eta_{3}$ liés aux intégrales sont nuls dans leurs domaines respectifs de l'intégration. Ainsi on obtient :

$$
\left.2 \rho \frac{\partial^{2} \zeta_{3}}{\partial t^{2}}-\partial_{\alpha \beta} m_{\alpha \beta}-\partial_{\beta}\left(\bar{N}_{\alpha \beta} \partial_{\alpha}\left(\zeta_{3}+\theta\right)\right)=p_{3} \text { dans } \omega \times\right] 0, T[,
$$

et

$$
\left.\partial_{\beta} \bar{N}_{\alpha \beta}=0 \text { dans } \omega \times\right] 0, T[,
$$

on déduit que :

$$
\left.\partial_{\beta}\left(\bar{N}_{\alpha \beta} \partial_{\alpha}\left(\zeta_{3}+\theta\right)\right)=\bar{N}_{\alpha \beta} \partial_{\alpha \beta}\left(\zeta_{3}+\theta\right) \text { dans } \omega \times\right] 0, T[,
$$

et on obtient :

$$
\left.2 \rho \frac{\partial^{2} \zeta_{3}}{\partial t^{2}}-\partial_{\alpha \beta} m_{\alpha \beta}-\bar{N}_{\alpha \beta} \partial_{\alpha \beta}\left(\zeta_{3}+\theta\right)=p_{3} \text { dans } \omega \times\right] 0, T[.
$$


De même on obtient :

$$
\left.\bar{N}_{\alpha \beta} \nu_{\beta}-2 \tilde{h}_{\alpha}=0 \operatorname{sur} \gamma \times\right] 0, T[,
$$

donc :

$$
\left.\bar{N}_{\alpha \beta} \nu_{\beta}=2 h_{\alpha} \operatorname{sur} \gamma_{1} \times\right] 0, T[,
$$

et

$$
\left.\bar{N}_{\alpha \beta} \nu_{\beta}=0 \text { sur } \gamma_{2} \times\right] 0, T[.
$$

En plus, on trouve :

$$
\left.m_{\alpha \beta} \nu_{\alpha} \nu_{\beta}=0 \text { sur } \gamma_{2} \times\right] 0, T[,
$$

et

$$
\left.\left[\partial_{\alpha} m_{\alpha \beta}+\bar{N}_{\alpha \beta} \partial_{\alpha}\left(\zeta_{3}+\theta\right)\right] \nu_{\beta}+\partial_{\tau}\left(m_{\alpha \beta} \nu_{\alpha} \tau_{\beta}\right)=0 \text { sur } \gamma_{2} \times\right] 0, T[
$$

et puisque $\bar{N}_{\alpha \beta} \nu_{\beta}=0$ sur $\left.\gamma_{2} \times\right] 0, T[$, on déduit que :

$$
\left.\partial_{\alpha} m_{\alpha \beta} \nu_{\beta}+\partial_{\tau}\left(m_{\alpha \beta} \nu_{\alpha} \tau_{\beta}\right)=0 \operatorname{sur} \gamma_{2} \times\right] 0, T[.
$$

\section{5. équivalence avec les équations de Marguerre-von Kármán généralisées dynamiques}

En suivant une démarche analogue à celle de [7] (voir aussi [6] pour les plaques de von Kármán généralisées), on établit le résultat suivant :

Théorème 5 Soit $\zeta=\left(\zeta_{i}\right)$ une solution du problème $(\bar{P}(\omega))$ avec la régularité : $\zeta_{\alpha} \in H^{3}(\omega), \zeta_{3} \in H^{4}(\omega) \forall t \in[0, T]$, alors

a) Les fonctions $\tilde{h}_{\alpha}$ sont dans l'espace $H^{\frac{3}{2}}(\gamma)$ et satisfaisants les conditions de compatibilité :

$$
\int_{\gamma} \tilde{h}_{1} d \gamma=\int_{\gamma} \tilde{h}_{2} d \gamma=\int_{\gamma}\left(x_{1} \tilde{h}_{2}-x_{2} \tilde{h}_{1}\right) d \gamma=0
$$

b) Il existe une fonction d'Airy $\Phi \in H^{4}(\omega)$, unique si l'on impose $\Phi(0)=\partial_{\alpha} \Phi(0)=0$, telle que :

$$
\left.\bar{N}_{11}=2 \partial_{22} \Phi, \bar{N}_{12}=\bar{N}_{21}=-2 \partial_{12} \Phi, \bar{N}_{22}=2 \partial_{11} \Phi \text { dans } \omega \times\right] 0, T[\text {. }
$$


c) Le couple $\left(\zeta_{3}, \Phi\right) \in H^{4}(\omega) \times H^{4}(\omega)$ vérifie les équations de Marguerre-von Kármán généralisées dynamiques :

$$
(P)\left\{\begin{array}{l}
\left.2 \rho \frac{\partial^{2} \zeta_{3}}{\partial t^{2}}+\frac{8 \mu(\lambda+\mu)}{3(\lambda+2 \mu)} \Delta^{2} \zeta_{3}=2\left[\Phi, \zeta_{3}+\theta\right]+p_{3} \text { dans } \omega \times\right] 0, T[, \\
\left.\Delta^{2} \Phi=-\frac{\mu(3 \lambda+2 \mu)}{2(\lambda+\mu)}\left[\zeta_{3}, \zeta_{3}+2 \theta\right] \text { dans } \omega \times\right] 0, T[, \\
\left.\zeta_{3}=\partial_{\nu} \zeta_{3}=0 \text { sur } \gamma_{1} \times\right] 0, T[, \\
\left.m_{\alpha \beta} \nu_{\alpha} \nu_{\beta}=0 \text { sur } \gamma_{2} \times\right] 0, T[ \\
\left.\partial_{\alpha} m_{\alpha \beta} \nu_{\beta}+\partial_{\tau}\left(m_{\alpha \beta} \nu_{\alpha} \tau_{\beta}\right)=0 \text { sur } \gamma_{2} \times\right] 0, T[, \\
\left.\Phi=\Phi_{0} \text { et } \partial_{\nu} \Phi=\Phi_{1} \text { sur } \gamma\right] 0, T[ \\
\zeta_{3}(., 0)=p_{3}^{0} \text { et } \frac{\partial \zeta_{3}}{\partial t}(., 0)=q_{3}^{0} \text { dans } \omega,
\end{array}\right.
$$

où :

$\Phi_{0}(y)=-y_{1} \int_{\gamma(y)} \tilde{h}_{2} d \gamma+y_{2} \int_{\gamma(y)} \tilde{h}_{1} d \gamma+\int_{\gamma(y)}\left(x_{1} \tilde{h}_{2}-x_{2} \tilde{h}_{1}\right) d \gamma$,

$\Phi_{1}(y)=-\nu_{1} \int_{\gamma(y)} \tilde{h}_{2} d \gamma+\nu_{2} \int_{\gamma(y)} \tilde{h}_{1} d \gamma, y=\left(y_{1}, y_{2}\right) \in \gamma$,

$[\Phi, \zeta]=\partial_{11} \Phi \partial_{22} \zeta+\partial_{22} \Phi \partial_{11} \zeta-2 \partial_{12} \Phi \partial_{12} \zeta$.

\section{Preuve.}

a) Par définition de $\bar{N}_{\alpha \beta}$, et puisque $\bar{N}_{\alpha \beta} \nu_{\beta}=2 \tilde{h}_{\alpha}$ sur $\gamma$, on déduit que :

$\tilde{h}_{\alpha} \in H^{\frac{3}{2}}(\gamma)$.

Les fonctions $\tilde{h}_{\alpha}$ satisfaisants les conditions de compatibilité (voir [6, Théorème 4]).

b) (i) De $\partial_{\beta} \bar{N}_{\alpha \beta}=0$ dans $\left.\omega \times\right] 0, T[$, et d'après le théorème de Poincaré généralisé (voir [10, Théorème VI, p.59]), il existe des distributions $\psi_{\alpha} \in D^{\prime}(\omega)$, uniques à une constante additive près, telle que :

$$
\begin{gathered}
\bar{N}_{11}=2 \partial_{2} \psi_{1}, \bar{N}_{12}=2 \partial_{2} \psi_{2}, \\
\bar{N}_{21}=-2 \partial_{1} \psi_{1}, \bar{N}_{22}=-2 \partial_{1} \psi_{2} .
\end{gathered}
$$

(ii) Puisque $\bar{N}_{12}=\bar{N}_{21}$, on déduit que $\partial_{\alpha} \psi_{\alpha}=0$, et d'après le théorème de Poincaré généralisé, il existe une distribution $\Phi \in D^{\prime}(\omega)$, unique à l'addition de polynômes de degré $\leq 1$ près, telle que :

$$
\psi_{1}=\partial_{2} \Phi, \psi_{2}=-\partial_{1} \Phi .
$$

Alors :

$\bar{N}_{11}=2 \partial_{22} \Phi, \bar{N}_{12}=\bar{N}_{21}=-2 \partial_{12} \Phi, \bar{N}_{22}=2 \partial_{11} \Phi$ dans $\left.\omega \times\right] 0, T[$.

De $\bar{N}_{\alpha \beta} \in H^{2}(\omega), \Phi(0)=\partial_{\alpha} \Phi(0)=0$ et puisque $\omega$ est un ouvert de Nikodym, il existe une fonction d'Airy $\Phi \in H^{4}(\omega)$ unique (voir [2, Théorème 5.1]). 
c) (i) $\left.\operatorname{De} \bar{N}_{\alpha \beta} \nu_{\beta}=2 \tilde{h}_{\alpha} \operatorname{sur} \gamma \times\right] 0, T[$, on obtient :

$$
\begin{gathered}
\tilde{h}_{1}=\frac{1}{2} \bar{N}_{1 \beta} \nu_{\beta}=\partial_{\tau}\left(\partial_{2} \Phi\right), \\
\tilde{h}_{2}=\frac{1}{2} \bar{N}_{2 \beta} \nu_{\beta}=-\partial_{\tau}\left(\partial_{1} \Phi\right),
\end{gathered}
$$

donc pour tout $y \in \gamma$, on trouve :

$$
\partial_{1} \Phi(y)=-\int_{\gamma(y)} \tilde{h}_{2} d \gamma \text { et } \partial_{2} \Phi(y)=\int_{\gamma(y)} \tilde{h}_{1} d \gamma
$$

de sorte que :

$$
\begin{aligned}
& \partial_{\nu} \Phi(y)=-\nu_{1}(y) \int_{\gamma(y)} \tilde{h}_{2} d \gamma+\nu_{2}(y) \int_{\gamma(y)} \tilde{h}_{1} d \gamma \\
& \partial_{\tau} \Phi(y)=-\tau_{1}(y) \int_{\gamma(y)} \tilde{h}_{2} d \gamma+\tau_{2}(y) \int_{\gamma(y)} \tilde{h}_{1} d \gamma .
\end{aligned}
$$

Alors :

$$
\left.\Phi=\Phi_{0} \text { et } \partial_{\nu} \Phi=\Phi_{1} \text { sur } \gamma \times\right] 0, T[
$$

(ii) Puisque $-\partial_{\alpha \beta} m_{\alpha \beta}=\frac{8 \mu(\lambda+\mu)}{3(\lambda+2 \mu)} \Delta^{2} \zeta_{3}, \bar{N}_{\alpha \beta} \partial_{\alpha \beta}\left(\zeta_{3}+\theta\right)=2\left[\Phi, \zeta_{3}+\theta\right]$, on déduit que:

$$
\left.2 \rho \frac{\partial^{2} \zeta_{3}}{\partial t^{2}}+\frac{8 \mu(\lambda+\mu)}{3(\lambda+2 \mu)} \Delta^{2} \zeta_{3}=2\left[\Phi, \zeta_{3}+\theta\right]+p_{3} \text { dans } \omega \times\right] 0, T[.
$$

(iii) De $\Delta^{2} \Phi=\frac{1}{2} \Delta \bar{N}_{\alpha \alpha}$ et $\partial_{\alpha \beta} \bar{N}_{\alpha \beta}=0$, on trouve :

$$
\left.\Delta^{2} \Phi=-\frac{\mu(3 \lambda+2 \mu)}{2(\lambda+\mu)}\left[\zeta_{3}, \zeta_{3}+2 \theta\right] \text { dans } \omega \times\right] 0, T[.
$$




\section{Conclusions et commentaires}

L'application de la méthode des développements asymptotiques aux équations élastodynamiques dans le cadre non linéaire d'une coque mince peu-profonde, considérée comme un milieu tri-dimensionnel, avec des conditions aux limites sur le bord latéral de type von Kármán généralisées (pression sur une partie du bord et le reste est libre), montre que le premier ordre significatif du développement asymptotique est solution d'un problème dynamique bi-dimensionnel non linéaire qui dépend de la fonction d'Airy $\Phi$ et de la déflexion $\zeta_{3}$.

Naturellement, on retrouve les équations de Marguerre-von Kármán classiques dynamiques en prenant $\gamma_{2}=\varnothing$. Si $\theta \equiv 0$ dans $\bar{\omega}$, la coque peu-profonde devient une plaque et les équations de Marguerre-von Kármán généralisées dynamiques se réduisent aux équations de von Kármán généralisées dynamiques.

Comme perspectives de ce travail, on se propose d'étudier l'existence des solutions du problème bi-dimensionnel $(P)$, représentant les équations de Marguerre-von Kármán généralisées dynamiques et la modélisation asymptotique du problème de Signorini (avec frottement) associé au problème $\left(\hat{P}^{\varepsilon} \cdot C\right)$.

\section{Bibliographie}

[1] P.G. Ciarlet, «A justification of the von Kármán equations », Arch. Rational Mech. Anal, vol. 73, 349-389, 1980.

[2] P.G. Ciarlet, J.C. Paumier, « A justification of the Marguerre-von Kármán equations », Comput. Mech, vol. 1, 177-202, 1986.

[3] P.G. Ciarlet, «Plates and junctions in elastic multistructures », Masson, 1990.

[4] P.G. Ciarlet, « Mathematical Elasticity, vol II, Theory of Plates », North-Holland, Amsterdam, 1997.

[5] G. ANDREOIU-BANICA, «Justification of the Marguerre-von Kármán equations in curvilinear coordinates », Asymptotic Anal, vol. 19, 35-55, 1999.

[6] P.G. Ciarlet, L. Gratie, « Generalized von Kármán equations », J. Math. Pures Appl., vol. 80(3), 263-279, 2001.

[7] L. GRATIE, « Generalized Marguerre-von Kármán equations of a nonlinearly elastic shallow shell », Applicable Anal, vol. 81, 1107-1126, 2002.

[8] P.G. Ciarlet, L. Gratie, «From the classical to the generalized von Kármán and Marguerrevon Kármán equations », Computational and Applied Mathematics, vol. 190, 470-486, 2006.

[9] P.G. Ciarlet, L. GratiE, « On the existence of solutions to the generalized Marguerre-von Kármán equations », Math. Mech. Solids, vol. 11, 83-100, 2006.

[10] L. SchwarTZ, « Théorie des distributions », Hermann, Paris, 1966.

[11] L.M. XIAO, «Asymptotic analysis of dynamic problems for linearly elastic shellsjustification of equations for dynamic membrane shells », Asymptotic Anal, vol. 17, 121-134, 
1998.

[12] L.M. XIAO, «Asymptotic analysis of dynamic problems for linearly elastic shellsjustification of equations for dynamic flexural shells », Chin. Ann. of Math., vol. 22B : 1, 13-22, 2001.

[13] L.M. XIAO, «Asymptotic analysis of dynamic problems for linearly elastic shellsjustification of equations for dynamic Koiter shells », Chin. Ann. of Math., vol. 22B : 3, 267-274, 2001.

[14] YE JI, «Asymptotic analysis of dynamic problem for linearly elastic generalized membrane shells », Asymptotic Anal, vol. 36, 47-62, 2003.

[15] YAN GUAN, «Asymptotic analysis of linearly elastodynamic shallow shells with variable thickness », Asymptotic Anal, vol. 50, 1-12, 2006.

[16] K. MARguerre, «Zur Theorie der gekrummten Platte grosser Formanderung », in : Proceedings, Fifth International Congress for Applied Mechanics, pp. 93-101, 1938.

[17] T. VON KÁRMÁN, H.S. Tsien, « The buckling of spherical shells by external pressure », $J$. Aero. Sci, vol. 7, 43-50, 1939. 